\title{
The Skyrme-TQRPA calculations of electron capture on hot nuclei in pre-supernova environment
}

\author{
Alan A. Dzhioev, ${ }^{1, *}$ A. I. Vdovin, ${ }^{1,+}$ and Ch. Stoyanov ${ }^{2,+}$ \\ ${ }^{1}$ Bogoliubov Laboratory of Theoretical Physics, JINR, 141980 Dubna, Russia \\ ${ }^{2}$ Institute for Nuclear Research and Nuclear Energy, \\ boul. Tzarigradsko Shaussee 73, 1784 Sofia, Bulgaria

\begin{abstract}
We combine the thermal QRPA approach with the Skyrme energy density func-
\end{abstract} \\ tional theory (Skyrme-TQRPA) for modelling the process of electron capture on \\ nuclei in supernova environment. For a sample nucleus, ${ }^{56} \mathrm{Fe}$, the Skyrme-TQRPA \\ approach is applied to analyze thermal effects on the strength function of $\mathrm{GT}_{+}$ \\ transitions which dominate electron capture at $E_{e} \leq 30 \mathrm{MeV}$. Several Skyrme in- \\ teractions are used in order to verify the sensitivity of the obtained results to the \\ Skyrme force parameters. Finite-temperature cross sections are calculated and the \\ results are compared with those of the other model calculations.
}

\section{INTRODUCTION}

Now it is well established that weak-interaction processes with nuclei play an important role in the dynamics of the collapsing core of a massive star that leads to a supernova explosion [1, 2]. During the pre-collapse phase, the competition between electron capture (EC) and $\beta$-decay determines the electron-to-baryon ratio $\left(Y_{e}\right)$ in the pre-supernova star and hence its Chandrasekhar mass proportional to $Y_{e}^{2}$. When the collapse proceeds, electron capture reduces the number of electrons available for pressure support, while $\beta$-decay acts in opposite direction. Until the core reaches densities of $\rho \sim 10^{11} \mathrm{~g} \mathrm{~cm}^{-3}$, neutrinos produced in this reaction leave the star freely, carrying away energy and helping to maintain a low entropy. As a result, nucleons resides primarily in nuclei. However, with increasing densities neutrino interactions with matter become important and influence the energy transfer from the core to the outer layers. So, the supernova simulations should include all potentially

*dzhioev@theor.jinr.ru

$\dagger$ vdovin@theor.jinr.ru

$\ddagger$ stoyanov@inrne.bas.bg 
important weak-interaction processes and reliable estimates of these rates and cross sections would contribute to a better understanding of the explosion mechanism.

In the present work, we focus our attention on electron capture. In the stellar environment electron energies are typically less than $30 \mathrm{MeV}$ and at early stage of collapse EC is dominated by Gamow-Teller $\left(\mathrm{GT}_{+}\right)$transitions in iron-group nuclei $(A=45-65)$. Therefore the Gamow-Teller strength functions in iron-group nuclei are of special importance. The task is complicated by the fact that under extreme conditions that hold in the supernova environment, $\mathrm{GT}_{+}$transitions from thermally-populated excited states of the parent nucleus may contribute significantly to EC. Unfortunately, to obtain information about GT + transitions from excited states in the terrestrial laboratory is not possible. Therefore, to describe EC probabilities in supernovae we should rely on theoretical (model) calculations.

Presently, the most reliable EC calculations for iron-group nuclei are performed by using large-scale shell-model (LSSM) diagonalization approach [3, 4]. For iron-group nuclei, present state-of-the-art shell model calculations provides a detailed GT strength distribution for the nuclear ground and excited states. However, for typical supernova temperatures $T \approx 1 \mathrm{MeV}$ too many states can be thermally populated and this makes state-by-state evolution of the individual $\mathrm{GT}_{+}$strength distributions computationally unfeasible. To overcome this problem the Brink hypothesis is applied, i.e., it is assumed that $\mathrm{GT}_{+}$strength distributions on nuclear excited states are the same as for the nuclear ground state. Thermal effects are treated by the so-called back-resonance contribution (see [4] for more details). However, the validity of Brink hypothesis for the $\mathrm{GT}_{+}$strength function is not obvious and even more the shell-model Monte-Carlo studies performed at finite temperatures [5] and the recent shell-model calculations [6] showed that the hypothesis is failed.

To predict EC rates and cross sections for hot nuclei, a so-called thermal quasiparticle random-phase approximation (TQRPA) was proposed recently in the framework of a statistical approach to the nuclear many-body problem at finite temperature [7, 8]. In this approach, rather than computing individual strength distributions for the nuclear ground and excited states, one determines an "average" temperature dependent strength function. In [7, 8], calculations were performed for ${ }^{54,56} \mathrm{Fe}$ and for neutron-rich germanium isotopes. The latter can be considered as the average nucleus at later stages of collapse [9]. It was found that the TQRPA does not support the Brink hypothesis and leads to noticeable thermal effects on the $\mathrm{GT}_{+}$strength function. As a result, for the Ge isotopes the low-energy 
cross sections are sensitive to temperature. Later on, the method was also applied to study neutrino-nucleus reactions in supernova environments [10, 11] and similar thermal effects on the low-energy cross section were found.

In [7, 8], the TQRPA calculations were based on the Hamiltonian of the QuasiparticlePhonon model (QPM) [12] with a phenomenological Saxon-Woods mean-field potential and schematic particle-hole interactions. The parameters of the QPM Hamiltonian were adjusted locally, i.e., to properties of a nucleus under consideration. This feature strongly reduces the predictive power of the theory.

In this paper, we extend our studies and perform self-consistent calculations combining the TQRPA approach with the Skyrme energy density functional theory. Use of the Skyrme forces makes more reliable theoretical predictions of the nuclear properties far from stability valley which play an important role in the process of stellar collapse.

The present calculations are performed within the finite-rank separable approximation, which expands the Skyrme residual interaction into a sum of separable terms in a systematic manner [13 15]. The factorization considerably reduces the computational effort of the TQRPA while maintaining high accuracy and even allows one to go beyond the TQRPA. It should be mentioned that in Refs. [16, 17] a finite-temperature RPA (FTRPA) model based on Skyrme functionals has been already applied to study EC in supernovae. Moreover, a similar approach, extended to the relativistic framework (FTRRPA), has been employed in Ref. [18]. However, in the cited papers thermal effects are treated not quite consistently. Below we discuss the subject in more details and compare our results with those of Refs. [16 18].

The paper is organized as follows. In Sec. II, we briefly outline the TQRPA formalism and the method of separabilization of the Skyrme residual interaction. In Sec. III, the GT thermal strength functions and electron capture cross sections are presented for the sample nuclei ${ }^{56} \mathrm{Fe}$. The results are compared with those obtained with the QPM Hamiltonian and within the FTRPA and FTRRPA frameworks. In Sec. IV, we draw conclusions and give an outlook for future studies. The derivation of the charge-exchange TQRPA equations for the finite-rank separable Skyrme interaction is given in Appendix A. 


\section{FORMALISM}

\section{A. Thermal strength function}

During the core-collapse phase of a supernova explosion the temperature in the core is sufficiently high (a few $10^{9} \mathrm{~K}$ ) to establish an equilibrium of reactions mediated by the strong and electromagnetic interactions [2]. Neglecting weak-interaction processes, one can consider nuclei as open quantum systems in thermal equilibrium with the heat and particle reservoir and, hence, they can be described as a thermal grand canonical ensemble with temperature $T$ and proton and neutron chemical potentials $\lambda_{p}$ and $\lambda_{n}$, respectively. Following Refs. [9, 19], to study EC on a hot nucleus we introduce a thermal strength function as a grand canonical average of transition matrix elements of the $\mathrm{GT}_{+}$operator between states $i$ and $f$ in the parent and daughter nuclei

$$
S_{\mathrm{GT}_{+}}(E, T)=\sum_{Z, N} \sum_{i, f} S_{i f}\left(\mathrm{GT}_{+}\right) \delta\left(E-E_{i f}\right) P\left(i, A_{N}^{Z}\right)
$$

Here, $S_{i f}\left(\mathrm{GT}_{+}\right)=\left|\left\langle f, A_{N+1}^{Z-1}\left|\sigma t_{+}\right| i, A_{N}^{Z}\right\rangle\right|^{2}$ and $E_{i f}=E_{f}-E_{i}+Q$ are, respectively, the transition strength and the transition energy, while $P\left(i, A_{N}^{Z}\right)$ determines the probability to find the initial state $i$ in the grand canonical ensemble. The $Q$ value is the difference between the masses of the daughter and parent nuclei, $Q=M_{d}-M_{p}$, and it determines the reaction threshold at $T=0$. Transition energy corresponds to the energy difference between the incoming electron and the outgoing neutrino, $E_{i f}=E_{e}-E_{\nu}$. At finite temperature, $E_{i f}$ can take negative values due to transitions from higher energy thermally excited states to lower energy states.

For the EC cross section one has

$$
\begin{aligned}
\sigma\left(E_{e}, T\right) & =\frac{\left(G_{F} g_{A}\right)^{2}}{2 \pi} F\left(Z, E_{e}\right) \int\left(E-E_{e}\right)^{2} S_{\mathrm{GT}_{+}}(E, T) d E \\
& =\sigma_{\mathrm{en}}\left(E_{e}, T\right)+\sigma_{\mathrm{ex}}\left(E_{e}, T\right)
\end{aligned}
$$

Here, $G_{F}$ is the weak interaction coupling constant, $g_{A}$ is the axial coupling constant, and $F\left(Z, E_{e}\right)$ is the Fermi function that accounts for the Coulomb distortion of the electron wave function near the nucleus (see, e.g., Ref. [4]). In Eq. (2), for further consideration, the total cross section is split into two parts: $\sigma_{\mathrm{en}}\left(E_{e}, T\right)$ describes the endoergic process which requires an energy input and includes only upward transitions $\left(E_{i f}>0\right)$, while $\sigma_{\text {ex }}\left(E_{e}, T\right)$ 
accounts for downward transitions $\left(E_{i f}<0\right)$ from thermally excited states and corresponds to the exoergic process when EC releases energy. At $T \neq 0$, the latter process is possible for arbitrary small incident electron energies, i.e., there is no reaction threshold at finite temperatures.

To compute the thermal strength function we apply the thermal quasiparticle randomphase approximation (TQRPA) which is based on the thermo-field dynamics (TFD) formalism. The concept of TFD is expounded in [20 22], and here we just briefly outline the key points relevant for the present discussion. In TFD, a hot nucleus is described by the state vector in the doubled Hilbert space which is a direct product of the original space and its isomorphic tilde space. Such doubling of the system degrees of freedom allows us to consider excitation and de-excitation processes at finite temperature. The correspondence between operators $A$ acting in the original Hilbert space and their tilde-partners $\widetilde{A}$ is given by the tilde-conjugation rules $[20-22]$. The important point is that the time evolution in the doubled Hilbert space is generated by the thermal Hamiltonian

$$
\mathcal{H}=H-\widetilde{H},
$$

where $\widetilde{H}=H\left(\widetilde{a}^{\dagger}, \widetilde{a}\right)$ is the tilde-partner of the original nuclear Hamiltonian $H\left(a^{\dagger}, a\right)$. The zero-energy eigenstate $|0(T)\rangle$ of the thermal Hamiltonian $\mathcal{H}$, which satisfies the thermal state condition

$$
A|0(T)\rangle=\sigma_{A} \mathrm{e}^{\mathcal{H} / 2 T} \widetilde{A}^{\dagger}|0(T)\rangle,
$$

is called the thermal vacuum and it describes the equilibrium state of a hot nucleus. Nonequilibrium states caused by an external perturbation correspond to non-zero energy eigenstates of the thermal Hamiltonian. By construction, the thermal Hamiltonian has both positiveand negative-energy (tilde) eigenstates, $\mathcal{H}|n\rangle=E_{n}|n\rangle$ and $\mathcal{H}|\widetilde{n}\rangle=-E_{n}|\widetilde{n}\rangle$.

Given the eigenstates of the thermal Hamiltonian, the thermal strength function for any transition operator $\mathcal{T}$ can be written as

$$
S_{\mathcal{T}}(E, T)=\sum_{n}\left\{S_{n}(\mathcal{T}) \delta\left(E-E_{n}\right)+\widetilde{S}_{n}(\mathcal{T}) \delta\left(E+E_{n}\right)\right\},
$$

where $S_{n}(\mathcal{T})$ and $\widetilde{S}_{n}(\mathcal{T})$ are the transition strengths from the thermal vacuum

$$
\begin{aligned}
S_{n}(\mathcal{T}) & =|\langle n|\mathcal{T}| 0(T)\rangle|^{2}, \\
\widetilde{S}_{n}(\mathcal{T}) & =|\langle\widetilde{n}|\mathcal{T}| 0(T)\rangle|^{2} .
\end{aligned}
$$


Obviously, in most practical cases one cannot diagonalize $\mathcal{H}$ exactly. In the present study, to obtain the thermal $\mathrm{GT}_{+}$strength function, we apply the TQRPA. In this method, nonequilibrium states of a hot nucleus are treated as phonon-like excitations on the thermal vacuum. Thus, the problem is reduced to the diagonalization of the thermal Hamiltonian in terms of phonon operators such that the respective phonon vacuum obeys the thermal-state condition (44). Below we briefly outline the method, while the details can be found in [8, 23] and in Appendix $\mathrm{A}$.

\section{B. Proton-neutron TQRPA with finite-rank separable approximation for the Skyrme interaction}

To obtain the thermal $\mathrm{GT}_{+}$strength function within the TQRPA we suppose that the nuclear proton and neutron Hartree-Fock states are already produced using the Skyrme energy density functional. In particular, it means that we ignore the influence of temperature on the nuclear mean field. Following [24], this stability of the mean field with respect to temperature is expected for $T$ considerably smaller than the energy difference between major shells $\left(\hbar \omega_{0}=41 A^{-1 / 3}\right)$. This requirement is well satisfied in nuclei with $A<100$ for the maximum temperatures reached during the collapse $(T \sim 5 \mathrm{MeV})$. Thus, the model Hamiltonian has the form

$$
H=H_{\mathrm{mf}}+H_{\mathrm{pair}}+H_{\mathrm{ph}}
$$

and it contains a spherical Skyrme-HF mean field for nucleons, the pairing interaction and the residual particle-hole interaction. Since we are working in the grand-canonical ensemble, the chemical potentials $\lambda_{n}$ and $\lambda_{p}$ are included into $H_{\mathrm{mf}}$. The particle-hole interaction $H_{\mathrm{ph}}$ is defined in terms of second derivatives of the Skyrme energy density functional with respect

to the one-body density [25] and can be written in terms of the Landau-Migdal theory of Fermi systems. Keeping only $l=0$ terms in $H_{\mathrm{ph}}$, the isovector part of the residual interaction which is responsible for charge-exchange excitations reads

$$
H_{\mathrm{ph}}=N_{0}^{-1}\left[F_{0}^{\prime}+G_{0}^{\prime} \sigma_{1} \cdot \sigma_{2}\right] \tau_{1} \cdot \tau_{2} \delta\left(\mathbf{r}_{1}-\mathbf{r}_{2}\right)
$$

where $\sigma$ and $\tau$ are the nucleon spin and isospin operators, and $N_{0}=2 k_{F} m^{*} / \pi^{2} \hbar^{2}$ with $k_{F}$ and $m^{*}$ denoting the Fermi momentum and nucleon effective mass, respectively. The expressions for the Landau parameters $F_{0}^{\prime}, G_{0}^{\prime}$ in terms of the Skyrme force parameters can 
be found in Ref. [26]. Here we just mention that due to the density dependence of the Skyrme interaction, the Landau parameters are functions of the coordinate $\mathbf{r}$.

Following the method presented in [13, 14], we apply an $N$-point integration Gauss formula and reduce the part of $H_{\mathrm{ph}}$ responsible for charge-exchange excitations to a finite-rank separable form

$$
H_{\mathrm{ph}}^{\mathrm{ch}}=-2 \sum_{k=1}^{N} \sum_{J M} \kappa_{F}^{(k)} M_{J M}^{(k) \dagger} M_{J M}^{(k)}-2 \sum_{k=1}^{N} \sum_{L J M} \kappa_{G}^{(k)} S_{L J M}^{(k) \dagger} S_{L J M}^{(k)} .
$$

The isovector interaction strengths, $\kappa_{F}^{(k)}$ and $\kappa_{G}^{(k)}$, are expressed via the Landau parameters [13, 14]. The multipole and spin-multipole operators entering $H_{\mathrm{ph}}$ are given by 1

$$
\begin{aligned}
\hat{M}_{J M}^{(k) \dagger} & =\hat{J}^{-1} \sum_{j_{p} j_{n}} f_{j_{p} j_{n}}^{(J k)}\left[a_{j_{p}}^{\dagger} a_{\overline{J_{n}}}\right]_{M}^{J}, \\
\hat{S}_{J M}^{(k) \dagger} & =\hat{J}^{-1} \sum_{j_{p} j_{n}} g_{j_{p} j_{n}}^{(L J k)}\left[a_{j_{p}}^{\dagger} a_{\overline{J_{n}}}\right]_{M}^{J},
\end{aligned}
$$

where $\hat{J}=\sqrt{2 J+1}$ and $f_{j_{p} j_{n}}^{(J k)}, g_{j_{p} j_{n}}^{(L J k)}$ denote the reduced single-particle matrix elements

$$
\begin{aligned}
f_{j_{p} j_{n}}^{(J k)} & =u_{j_{p}}\left(r_{k}\right) u_{j_{n}}\left(r_{k}\right)\left\langle j_{p}\left\|i^{J} Y_{J}\right\| j_{n}\right\rangle \\
g_{j_{p} j_{n}}^{(L J k)} & =u_{j_{p}}\left(r_{k}\right) u_{j_{n}}\left(r_{k}\right)\left\langle j_{p}\left\|i^{L}\left[Y_{L} \times \sigma\right]_{J}^{M}\right\| j_{n}\right\rangle .
\end{aligned}
$$

The radial wave functions $u_{j}\left(r_{k}\right)$ are related to the Hartree-Fock single-particle wave functions [13, 14], while $r_{k}$ are abscissas used in the $N$-point integration Gauss formula.

Following the TFD, to study charge-exchange excitations in a hot nucleus we should double the original nuclear degrees of freedom by introducing the tilde creation and annihilation operators $\widetilde{a}_{j m}^{\dagger}, \widetilde{a}_{j m}$ and then diagonalize the respective thermal Hamiltonian (3). Within the TQRPA, the thermal Hamiltonian is diagonalized in two steps. First, we introduce thermal quasiparticles that diagonalize the mean field and pairing parts of $\mathcal{H}$

$$
\mathcal{H}_{\mathrm{mf}}+\mathcal{H}_{\mathrm{pair}} \simeq \sum_{\tau=n, p} \sum_{j m}^{\tau} \varepsilon_{j m}(T)\left(\beta_{j m}^{\dagger} \beta_{j m}-\widetilde{\beta}_{j m}^{\dagger} \widetilde{\beta}_{j m}\right) .
$$

Thermal quasiparticles account pairing correlations at finite temperature and their energy and structure are found from the finite temperature BCS equations (see. Ref. [8] for more details). In accordance with the BCS theory [27, 28], the numerical solution of these equations yields vanishing of pairing correlations above a certain critical temperature $T_{\mathrm{cr}}$.

\footnotetext{
${ }^{1}$ In (10) and hereinafter, [ $]_{M}^{J}$ denotes the coupling of two single-particle angular momenta $j_{p}, j_{n}$ to the angular momentum $J$. The bar over index $j$ implies time inversion.
} 
The next step is to account for the residual particle-hole interaction and diagonalize the thermal Hamiltonian in terms of thermal phonon creation and annihilation operators

$$
\mathcal{H} \simeq \sum_{J M i} \omega_{J i}(T)\left(Q_{J M i}^{\dagger} Q_{J M i}-\widetilde{Q}_{J M i}^{\dagger} \widetilde{Q}_{J M i}\right)
$$

The energies and structure of thermal charge-exchange phonons are obtained by the solution of TQRPA equations. The explicit form of the proton-neutron TQRPA equations for the finite-rank separable Skyrme forces is given in the Appendix.

Charge-exchange $\mathrm{GT}_{+}$transitions from the thermal vacuum result in $J^{\pi}=1^{+}$thermal phonon states. Once the energies and structure of $1^{+}$thermal phonons are determined, one can evaluate the thermal strength function (5) for GT + transitions. The transition strengths are given by the following reduced matrix elements 2

$$
\begin{aligned}
& S_{i}\left(\mathrm{GT}_{+}\right)=\left|\left\langle Q_{1^{+} i}\left\|\sigma t_{+}\right\| 0(T)\right\rangle\right|^{2}, \\
& \widetilde{S}_{i}\left(\mathrm{GT}_{+}\right)=\left|\left\langle\widetilde{Q}_{1^{+} i}\left\|\sigma t_{+}\right\| 0(T)\right\rangle\right|^{2},
\end{aligned}
$$

while the respective transition energies are

$$
\begin{aligned}
& E_{i}=\omega_{J i}+\delta_{n p}, \\
& \widetilde{E}_{i}=-\omega_{J i}+\delta_{n p},
\end{aligned}
$$

where $\delta_{n p}=\lambda_{n}-\lambda_{p}+\Delta M_{n p}$, and $\Delta M_{n p}=1.29 \mathrm{MeV}$ is the neutron-proton mass difference (the contribution $\delta_{n p}$ arises because in charge-exchange reactions the initial and final nucleons are attached to different nucleon systems). Thus, within the TQRPA we have both positiveand negative-energy transitions to thermal phonon states. The latter contribute to exoergic EC when $E_{\nu}>E_{e}$. It should be emphasized that in the zero-temperature limit, transition strengths to tilde-phonon states vanish and the TQRPA method reduces into the standard QRPA. In particular, at $T=0$ the transition energies $E_{i}=\omega_{J i}+\lambda_{n}-\lambda_{p}+\Delta M_{n p}$ correspond to the excitation energies with respect to the parent nucleus ground state.

In concluding this section we would like to point out that the thermal strength function for $\mathrm{GT}_{-}$transitions can be obtained by the same method. In [7], it was shown that within the TQRPA the total $\mathrm{GT}_{-}$and $\mathrm{GT}_{+}$strengths fulfill the Ikeda sum rule

$$
S_{-}-S_{+}=3(N-Z)
$$

\footnotetext{
${ }^{2}$ Explicit expressions for transition strengths can be found in [7, 8]
} 
where $S_{\mp}=\int S_{\mathrm{GT}_{\mp}} d E$. Moreover, the $\mathrm{GT}_{-}$and $\mathrm{GT}_{+}$strength functions are related by the detailed balance

$$
S_{\mathrm{GT}_{-}}(-E, T)=S_{\mathrm{GT}_{+}}(E, T) \exp \left\{-\frac{E-\delta_{n p}}{T}\right\} .
$$

Thus, for each $n \rightarrow p(p \rightarrow n)$ GT transition with energy $E>0$ there is an inverse $p \rightarrow n$ $(n \rightarrow p)$ transition with energy $-E$ and the respective transition strengths are connected by (17). In [11], we have shown in a model-independent way that the relation (17) is valid in the grand-canonical ensemble for any transition operators $\mathcal{T}_{-}$and $\mathcal{T}_{+}$, which differ only by the isospin operator.

\section{RESULTS}

In this section, we employ the theoretical framework described above to compute EC cross sections on ${ }^{56} \mathrm{Fe}$ at finite $T$. Experimental data available for this nucleus allow to test our calculations at zero temperature. Besides, EC calculations for ${ }^{56} \mathrm{Fe}$ in the supernova environment have been performed within various theoretical approaches [16 18] and these results can be compared with those of the TQRPA. To reveal the sensitivity of the results to the Skyrme interaction parametrization, we perform the calculations for a representative set of Skyrme forces: Sly4 [29], SGII [26], and SkM* [30]. To distinguish the present results from those obtained with the QPM Hamiltonian [8, 11], we will refer to them as the SkyrmeTQRPA and the QPM-TQRPA, respectively.

A short comment should be made concerning the choice of the pairing interaction. Within the BCS approach the phase transition in nuclei from the superfluid to normal state occurs at critical temperature $T_{\text {cr }} \approx 0.5 \Delta$, where $\Delta$ is the ground state pairing gap [27, 28]. Therefore, the inclusion of particle-particle residual interactions does not affect the strength function for temperatures $T>T_{\text {cr }}$. However, to compute the ground state $\mathrm{GT}_{+}$distributions and compare them with the experimental and shell-model ones, pairing correlations are taken into account at zero temperature. As in [8, 11] we employ presently a BCS Hamiltonian with a constant pairing strength. The neutron and proton pairing strength parameters are fixed to reproduce the odd-even mass difference. At $T=0$ the resulting proton and neutron energy gaps for ${ }^{56} \mathrm{Fe}$ are $\Delta_{p}=1.57 \mathrm{MeV}$ and $\Delta_{n}=1.36 \mathrm{MeV}$, respectively. Thus, the critical temperature when the pairing phase transition occurs is $T_{\text {cr }} \approx 0.8 \mathrm{MeV}$. 


\section{A. GT + strength function at zero and finite temperatures}

In this subsection, we discuss temperature evolution of the $\mathrm{GT}_{+}$strength function in ${ }^{56} \mathrm{Fe}$. To begin with, let us first consider the results of QRPA calculations at zero temperature. In Fig. 1, we show the ground state $\mathrm{GT}_{+}$strength distribution, whose measurement is feasible from $(n, p)$ [31] reactions on the ${ }^{56} \mathrm{Fe}$ target. Notice that all distributions are plotted as functions of the excitation energy with respect to the parent nucleus ground state. The experimental data from Ref. [31] are indicated by points and for convenience of comparison with the QRPA results they are multiplied by a factor of 5. The GT + centroid energy, 6.81 MeV, predicted by the LSSM calculations [3] is shown by an arrow (to obtain this number we have added the mass splitting between daughter and parent nucleus, $M\left({ }^{56} \mathrm{Mn}\right)-$ $M\left({ }^{56} \mathrm{Fe}\right)=4.21 \mathrm{MeV}$, to the number in Table 1 of Ref. [3] $)$.

Looking at Fig. 1 1 one can see that the structure of the GT + strength distributions is qualitatively similar for all the Skyrme forces. Namely, our Skyrme-QRPA calculations produce strength distributions mainly concentrated in a single resonance peak. The peak is dominated by the single-particle transition $\pi 1 f_{7 / 2} \rightarrow \nu 1 f_{5 / 2}$. Although the resonance is displaced in energy for the different Skyrme interactions, the deviation of the main theoretical peak from the maximum of experimental strength distribution lies within $1 \mathrm{MeV}$. When comparing the resonance energy with the LSSM results, we notice that the QRPA calculations with SLy4 and SkM* fairly well reproduce the $\mathrm{GT}_{+}$energy centroid predicted by the shell-model calculations. Of course, the simple QRPA calculations cannot reproduce the fragmentation of the strength, that is, the spreading width. In this respect the LSSM calculations [3] are clearly advantageous.

Figure 1also shows the unperturbed $\mathrm{GT}_{+}$strength distributions obtained within the BCS approach, i.e., neglecting the particle-hole residual interaction $H_{\mathrm{ph}}$. As evident from the figure, the particle-hole interaction pushes the $\mathrm{GT}_{+}$strength to higher energies and the energy shift is the greatest for the QPM-QRPA calculations. Moreover, due to particle-hole correlations the $\mathrm{GT}_{+}$strength distribution calculated within QPM-QRPA is more fragmented. At the same time, the BCS and QRPA calculations with the SLy4 force produce practically the same strength distributions. It means that for the SLy4 Skyrme force the p-h residual interaction in spin-isospin channel is very weak. Not only the resonance energy, but also the total $\mathrm{GT}_{+}$strength $S_{+}$is affected by the residual interaction. Within the BCS, all cal- 
culations predict rather close values of $S_{+} \approx 10 \div 11$. The particle-hole correlations reduce the total $\mathrm{GT}_{+}$strength and this effect is most significant for the QPM based calculations. However, despite the reduction, the QRPA values of $S_{+}$noticeably overestimate the experimental ones $\left(S_{+}=2.9 \pm 0.3[\underline{31}]\right)$. The experimentally observed quenching of the total GT strength is usually reproduced by reducing the axial coupling constant from its free-nucleon value $g_{A}=-1.26$ to some effective value $g_{A}^{*}$. In what follows we will use $g_{A}^{*}=-0.93$, that corresponds to renormalization of the GT matrix elements by a quenching factor 0.74 . The same quenching factor was used in the shell-model calculations [3].

Let us now compare the results of Ref. [8, 11] where thermal effects on the $\mathrm{GT}_{+}$strength function where studied within the QPM-TQRPA approach with the present self-consistent scheme based on the Skyrme energy density functional theory. The GT + thermal strength function in ${ }^{56} \mathrm{Fe}$ is shown in Fig. 2 at $T=1 \mathrm{MeV}$. To make the thermal effects clearly defined the ground state $(T=0)$ strength functions are also shown. Note, that the strength functions are displayed in a logarithmic scale.

In Fig. 2, one can easily see that the Brink hypothesis is not valid for hot nuclei and the $\mathrm{GT}_{+}$strength function evolves with temperature. Effective interaction affects this thermal effect quantitatively but not qualitatively. For the upward $(E>0)$ strength, the main effect is a temperature-induced shift of the $\mathrm{GT}_{+}$resonance towards lower energies. This decrease is mainly attributed to the vanishing of pairing correlations, since at temperatures above the critical one no extra energy is needed to break a proton Cooper pair when performing $\mathrm{GT}_{+}$transitions. Our QPM-TQRPA and Skyrme-TQRPA calculations show that when the temperature is increased up to $1 \mathrm{MeV}$, the $\mathrm{GT}_{+}$resonance is lowered by about $1.5 \mathrm{MeV}$. In particular, calculations with the SGII force demonstrate that due to pairing collapse the $\mathrm{GT}_{+}$resonance shifts below the ground-state reaction threshold $Q=M\left({ }^{56} \mathrm{Mn}\right)-M\left({ }^{56} \mathrm{Fe}\right)$. However, not only vanishing of pairing correlations causes the resonance downward shift. It was shown in [11], that owing to the thermal blocking of the residual interaction, a further increase in temperature could decrease the $\mathrm{GT}_{+}$resonance as well. As mentioned in the Introduction, the observed temperature-induced downward shift of the $\mathrm{GT}_{+}$resonance is not present in LSSM calculations, since they are partially based on the Brink hypothesis. In contrast, the finite temperature relativistic QRPA calculations [18] and shell-model MonteCarlo calculations [5] show similar features for the changes of the $\mathrm{GT}_{+}$resonance energy.

At finite temperature, $\mathrm{GT}_{+}$transitions which are Pauli blocked at $T=0$ due to closed 
neutron subshell become unblocked due to thermal smearing of the nuclear Fermi surface. Similarly, protons that are thermally excited to higher orbitals can undergo GT + transitions. In ${ }^{56} \mathrm{Fe}$, such thermally unblocked transitions lead to appearance of the downward $(E<0)$ component in the $\mathrm{GT}_{+}$strength function. It is interesting to note that both the QPMTQRPA and Skyrme-TQRPA calculations predict roughly the same energy region where the thermally unblocked $\mathrm{GT}_{+}$strength appears at $T=1 \mathrm{MeV}$. The single-particle transitions which mainly contribute to this strength are $\pi 2 p_{3 / 2} \rightarrow \nu 2 p_{3 / 2,1 / 2}$ particle-particle and $\pi 1 f_{7 / 2} \rightarrow \nu 1 f_{7 / 2}$ hole-hole transitions. Here particle (hole) denotes a state above (below) the Fermi level.

It should be emphasized that the appearance of downward transitions in the TQRPA thermal strength function stems from the doubling of the system degrees of freedom within the TFD. For ${ }^{56} \mathrm{Fe}$, this downward strength corresponds to transitions to tilde-phonon states, i.e., to negative-energy solutions of the TQRPA equations. No such negative-energy transitions appear within the approaches based on the finite temperature RPA used in [16 18]. Therefore, only upward $\mathrm{GT}_{+}$transitions were considered in calculations of the EC rates on ${ }^{56} \mathrm{Fe}$ within the FTRPA and FTRRPA.

\section{B. Electron capture cross section}

In Fig. 3, we display the calculated EC cross sections (2) as functions of the incident electron energy $E_{e}$. The cross sections are shown at three different temperatures, $T=$ 0.5, 1.0 and 2.0 MeV. Moreover, the Skyrme-TQRPA results are presented together with those of the QPM-TQRPA calculations. As seen from the plots, all models predict a universal behavior of the cross section versus electron energy and temperature. In particular, there is no reaction threshold for EC at finite temperature and the low-energy cross sections demonstrate a significant thermal enhancement. It is clear that both these effects are caused by downward $\mathrm{GT}_{+}$transitions from thermally excited states which contribution to the cross section increases with temperature.

The bottom panels of Fig. 3 show the ratio of exoergic EC to the reaction cross section

$$
\beta\left(E_{e}, T\right)=\frac{\sigma_{\mathrm{ex}}\left(E_{e}, T\right)}{\sigma\left(E_{e}, T\right)} .
$$

As expected, the ratio $\beta \sim 1$ for low-energy electrons and then gradually decreases with 
increasing electron energy. Moreover, the higher the temperature the wider is the range of $E_{e}$ when exoergic process dominates (i.e., $\left.\beta>0.5\right)$ EC. It should be stressed that all variants of the Skyrme forces used here give rather similar results. The spread in calculated cross sections is less than an order of magnitude at low energies and temperatures and it decreases with the increase of $T$ and $E_{e}$. The Skyrme-TQRPA calculations systematically predict cross sections above the values obtained within the QPM-TQRPA model. Evidently, the discrepancy reflects the differences in the total $\mathrm{GT}_{+}$strength (see $S_{+}$values in Fig. (1).

In Fig. 4 , the present results of the Skyrme-TQRPA calculations at $T=1 \mathrm{MeV}$ are compared with those obtained by the FTRPA [17] and the FTRRPA [18] calculations. In each plot we compare the TQRPA and FTRPA cross sections calculated with the same Skyrme force. One can notice in the figure that the FTRPA and FTRRPA calculations predict the cross section rapidly dropping to zero when the electron energy tends to some threshold value. As was pointed above, the FTRPA and FTRRPA approaches do not include downward $\mathrm{GT}_{+}$transitions that contribute to the exoergic EC. For this reason some minimal electron energy is required to trigger the EC process. In contrast, within the TQRPA, downward transitions dominate the low-energy cross section at $T=1.0 \mathrm{MeV}$ and make possible EC for arbitrary small incident electron energy.

In Fig. 4, we also display the endoergic component of the cross section calculated with the Skyrme-TQRPA. As seen, the general behavior of $\sigma_{\mathrm{en}}\left(E_{e}, T\right)$ as a function of $E_{e}$ is in agreement with the FTRPA and TQRPA calculations. However, the TQRPA results are much closer to those computed within the FTRRPA framework than to the FTRPA results obtained with the same Skyrme forces. Namely, the FTRPA cross sections are shifted to higher electron energies with respect to our $\sigma_{\text {en }}\left(E_{e}, T\right)$ and the shift is practically the same $(\sim 3 \mathrm{MeV})$ for all the Skyrme forces used. It seems that the discrepancy reflects the difference in the $\mathrm{GT}_{+}$peak position and the reason for this most likely lies in the different definition of transition energies. To explain this, we recall that in the FTRPA it is assumed 3 that the RPA energy corresponds to the excitation energy in the daughter nucleus. In such a case, one can approximately write the transition energy to the $\mathrm{GT}_{+}$resonance in ${ }^{56} \mathrm{Fe}$ as

$$
E_{\mathrm{FTRPA}}=E\left(\nu 1 f_{5 / 2}\right)-E\left(\pi 1 f_{7 / 2}\right)+\Delta H_{p h}+Q,
$$

where $Q=M\left({ }^{56} \mathrm{Mn}\right)-M\left({ }^{56} \mathrm{Fe}\right)=4.21 \mathrm{MeV}$ and $\Delta H_{p h}$ is the energy shift induced by the ${ }^{3}$ See Eq. (4) in Ref. [17] and discussion therein. 
residual interaction. In the TQRPA framework, the transition energy is defined by Eq. (15) and it can be written as

$$
\begin{aligned}
E_{\mathrm{TQRPA}} & =\varepsilon\left(\nu 1 f_{7 / 2}\right)+\varepsilon\left(\nu 1 f_{7 / 2}\right)+\Delta H_{p h}+\delta_{n p} \\
& =E\left(\nu 1 f_{5 / 2}\right)-E\left(\pi 1 f_{7 / 2}\right)+\Delta H_{p h}+\Delta M_{n p} .
\end{aligned}
$$

Here, we take into account that in the absence of pairing correlations single-particle and quasiparticle energies are connected as $\varepsilon= \pm(E-\lambda)$, where the upper sign is for particle states, and the lower sign is for hole states. Thus, one has $E_{\mathrm{FTRPA}}-E_{\mathrm{TQRPA}}=2.92 \mathrm{MeV}$ which is very close to the observed energy shift between the FTRPA cross section and our $\sigma_{\text {en }}\left(E_{e}, T\right)$. It should be also mentioned that under the FTRRPA (see Eq. (14) in Ref. [18]) the transition energy $E_{e}-E_{\nu}$ is determined by the same manner as under the TQRPA (at $T>T_{\text {cr }}$ ). Therefore, it is not surprising that the FTTRPA cross section is in a good agrement with our $\sigma_{\mathrm{en}}\left(E_{e}, T\right)$.

\section{SUMMARY AND CONCLUSIONS}

In the present work the electron capture cross sections on the hot ${ }^{56} \mathrm{Fe}$ nucleus were calculated in the supernova environment. The thermal effects were treated within the thermal QRPA combined with Skyrme energy density functional theory. The results were compared with those obtained from the TQRPA calculations with the QPM Hamiltonian as well as from the finite temperature RPA and relativistic RPA approaches (see Ref. [17] and Ref. [18], respectively).

We perform a detailed analysis of thermal effects on the $\mathrm{GT}_{+}$transitions which dominate the EC cross sections for $E_{e} \leq 30 \mathrm{MeV}$. It was found that the self-consistent TQRPA calculations with the Skyrme forces predict the same thermal effects on the $\mathrm{GT}_{+}$strength function as those found in our previous studies based on the QPM Hamiltonian. In particular, increasing temperature shifts the $\mathrm{GT}_{+}$resonance to lower energies and makes possible negative-energy transitions. The values of the resonance shift and the energies of thermally unblocked downward transitions well agree for all Skyrme-TQRPA calculations.

We calculate the EC cross sections for different supernova temperatures. The spread in the cross sections computed with the different Skyrme forces is less than an order of magnitude. This finding is the main result of the present study. Comparison with the 
FTRPA and FTRRPA results $[17,18]$ reveals the importance of downward GT $_{+}$transitions for the low-energy EC cross section.

The application of the present self-consistent method is not restricted by iron-group nuclei and it can be applied to more massive neutron-rich nuclei, which are beyond the present capability of the LSSM calculations. The fragmentation of the $\mathrm{GT}_{+}$strength plays a significant role at low temperature and densities of the supernova environment. Therefore, a further improvement of the model is to go beyond TQRPA and take into account higherorder correlations. For the separable residual interaction this can be done by coupling the thermal phonon states with more complex (e.g., two-phonon) configurations. For chargeexchange excitations at zero temperature, the phonon coupling was considered within the QPM model [32], and most recently with the self-consistent Skyrme based calculations [33]. Another possible improvement is the inclusion of the effect of nuclear deformation. In [34], EC calculations for deformed nuclei were performed assuming that the process is dominates by the ground-state contribution.

\section{ACKNOWLEDGMENTS}

We thank Dr. A. P. Severyukhin for fruitful discussions.

\section{Appendix A}

Within the TQRPA, charge-exchange thermal phonons are defined as a linear superposition of the proton-neutron thermal quasiparticle pair creation and annihilation operators

$$
\begin{aligned}
Q_{J M i}^{\dagger}=\sum_{j_{p} j_{n}}( & \psi_{j_{p} j_{n}}^{J i}\left[\beta_{j_{p}}^{\dagger} \beta_{j_{n}}^{\dagger}\right]_{M}^{J}+\widetilde{\psi}_{j_{p} j_{n}}^{J i}\left[\widetilde{\beta}_{\overline{\jmath_{p}}}^{\dagger} \widetilde{\beta}_{\overline{\jmath_{n}}}^{\dagger}\right]_{M}^{J}+i \eta_{j_{p} j_{n}}^{J i}\left[\beta_{j_{p}}^{\dagger} \widetilde{\beta}_{\overline{\jmath_{n}}}^{\dagger}\right]_{M}^{J}+i \widetilde{\eta}_{j_{p} j_{n}}^{J i}\left[\widetilde{\beta}_{\overline{J_{p}}}^{\dagger} \beta_{j_{n}}^{\dagger}\right]_{M}^{J} \\
& \left.+\phi_{j_{p} j_{n}}^{J i}\left[\beta_{\overline{\bar{J}_{p}}} \beta_{\overline{\bar{J}_{n}}}\right]_{M}^{J}+\widetilde{\phi}_{j_{p} j_{n}}^{J i}\left[\widetilde{\beta}_{j_{p}} \widetilde{\beta}_{j_{n}}\right]_{M}^{J}+i \xi_{j_{p} j_{n}}^{J i}\left[\beta_{\overline{\bar{J}_{p}}} \widetilde{\beta}_{j_{n}}\right]_{M}^{J}+i \widetilde{\xi}_{j_{p} j_{n}}^{J i}\left[\widetilde{\beta}_{j_{p}} \beta_{\overline{J_{n}}}\right]_{M}^{J}\right)
\end{aligned}
$$

The physical meaning of different terms in this definition is explained in [8, 11]. Here we just mention that due to negative-energy tilde thermal quasiparticles in (A1), the spectrum of thermal charge-exchange phonons contains negative-energy and low-energy states which do not exist at zero temperature. These "new" phonon states are interpreted as thermally unblocked transitions between nuclear excited states. 
To find the energy and the structure of the thermal phonons we apply the equation of motion method

$$
\left.\langle| \delta Q,\left[\mathcal{H}, Q^{\dagger}\right]\right]|\rangle=\omega(T)\left\langle\left|\left[\delta Q, Q^{\dagger}\right]\right|\right\rangle
$$

under two additional constraints: (a) the phonon operators obey Bose commutation relations, and (b) the phonon vacuum obeys the thermal state condition (4). The first constraint is equivalent to averaging with respect to the BCS thermal vacuum in the equation of motion and it leads to an orthonormality condition for the phonon amplitudes

$$
\begin{aligned}
& \sum_{j_{p} j_{n}}\left(\psi_{j_{p} j_{n}}^{J i} \psi_{j_{p} j_{n}}^{J i^{\prime}}+\widetilde{\psi}_{j_{p} j_{n}}^{J i} \widetilde{\psi}_{j_{p} j_{n}}^{J i^{\prime}}+\eta_{j_{p} j_{n}}^{J i} \eta_{j_{p} j_{n}}^{J i^{\prime}}+\widetilde{\eta}_{j_{p} j_{n}}^{J i} \widetilde{\eta}_{j_{p} j_{n}}^{J i^{\prime}}\right. \\
& \left.-\phi_{j_{p} j_{n}}^{J i} \phi_{j_{p} j_{n}}^{J i^{\prime}}-\widetilde{\phi}_{j_{p} j_{n}}^{J i} \widetilde{\phi}_{j_{p} j_{p}}^{J i^{\prime}}-\xi_{j_{p} j_{n}}^{J i} \xi_{j_{p} j_{n}}^{J i^{\prime}}-\widetilde{\xi}_{j_{p} j_{n}}^{J i} \widetilde{\xi}_{j_{p} j_{n}}^{J i^{\prime}}\right)=\delta_{i i^{\prime}} .
\end{aligned}
$$

The last assumption yields the following relations between amplitudes:

$$
\begin{aligned}
& \left(\begin{array}{c}
\widetilde{\psi} \\
\widetilde{\phi}
\end{array}\right)_{j_{p} j_{n}}^{J i}=\frac{y_{j_{p}} y_{j_{n}}-\mathrm{e}^{-\omega_{J i} / 2 T} x_{j_{p}} x_{j_{n}}}{\mathrm{e}^{-\omega_{J i} / 2 T} y_{j_{p}} y_{j_{n}}-x_{j_{p}} x_{j_{n}}}\left(\begin{array}{c}
\phi \\
\psi
\end{array}\right)_{j_{p} j_{n}}^{J i}, \\
& \left(\begin{array}{c}
\widetilde{\eta} \\
\widetilde{\xi}
\end{array}\right)_{j_{p} j_{n}}^{J i}=\frac{y_{j_{p}} x_{j_{n}}-\mathrm{e}^{-\omega_{J i} / 2 T} x_{j_{p}} y_{j_{n}}}{\mathrm{e}^{-\omega_{J i} / 2 T} y_{j_{p}} x_{j_{n}}-x_{j_{p}} y_{j_{n}}}\left(\begin{array}{c}
\xi \\
\eta
\end{array}\right)_{j_{p} j_{n}}^{J i} .
\end{aligned}
$$

Here, $x_{j}$ and $y_{j}\left(x_{j}^{2}+y_{j}^{2}=1\right)$ are the coefficients of the so-called thermal transformation which establishes a connection between Bogoliubov and thermal quasiparticles. Note that $y_{j}$ are given by the nucleon Fermi-Dirac function and they define a number of thermally excited Bogoliubov quasiparticles in the thermal vacuum (see [8] for more details).

To derive the TQRPA equations it is convenient to introduce the following linear combinations of amplitudes:

$$
\begin{array}{ll}
\left(\begin{array}{c}
g \\
w
\end{array}\right)_{j_{p} j_{n}}^{J i}=\psi_{j_{p} j_{n}}^{J i} \pm \phi_{j_{p} j_{m}}^{J i}, & \left(\begin{array}{c}
\widetilde{g} \\
\widetilde{w}
\end{array}\right)_{j_{p} j_{n}}^{J i}=\widetilde{\psi}_{j_{p} j_{n}}^{J i} \pm \widetilde{\phi}_{j_{p} j_{n}}^{J i}, \\
\left(\begin{array}{c}
t \\
s
\end{array}\right)_{j_{p} j_{n}}^{J i}=\eta_{j_{p} j_{n}}^{J i} \pm \xi_{j_{p} j_{n}}^{J i}, & \left(\begin{array}{c}
\widetilde{t} \\
\widetilde{s}
\end{array}\right)_{j_{p} j_{n}}^{J i}=\widetilde{\eta}_{j_{p} j_{n}}^{J i} \pm \widetilde{\xi}_{j_{p} j_{n}}^{J i} .
\end{array}
$$


Then from ( $\underline{\mathrm{A} 4})$ it follows that

$$
\begin{aligned}
& \left(\begin{array}{c}
g \\
w
\end{array}\right)_{j_{p} j_{n}}^{J i}=\left(x_{j_{p}} x_{j_{n}}-\mathrm{e}^{-\omega_{J i} / 2 T} y_{j_{p}} y_{j_{n}}\right)\left(\begin{array}{c}
G \\
W
\end{array}\right)_{j_{p} j_{n}}^{J i} \\
& \left(\begin{array}{c}
\widetilde{g} \\
\widetilde{w}
\end{array}\right)_{j_{p} j_{n}}^{J i}=\mp\left(y_{j_{p}} y_{j_{n}}-\mathrm{e}^{-\omega_{J i} / 2 T} x_{j_{p}} x_{j_{n}}\right)\left(\begin{array}{c}
G \\
W
\end{array}\right)_{j_{p} j_{n}}^{J i} \\
& \left(\begin{array}{c}
t \\
s
\end{array}\right)_{j_{p} j_{n}}^{J i}=\left(x_{j_{p}} y_{j_{n}}-\mathrm{e}^{-\omega_{J i} / 2 T} y_{j_{p}} x_{j_{n}}\right)\left(\begin{array}{c}
T \\
S
\end{array}\right)_{j_{p} j_{n}}^{J i} \\
& \left(\begin{array}{c}
\widetilde{t} \\
\widetilde{s}
\end{array}\right)_{j_{p} j_{n}}^{J i}=\mp\left(y_{j_{p}} x_{j_{n}}-\mathrm{e}^{-\omega_{J i} / 2 T} x_{j_{p}} y_{j_{n}}\right)\left(\begin{array}{c}
T \\
S
\end{array}\right)_{j_{p} j_{n}}^{J i},
\end{aligned}
$$

where $G, W, T$ and $S$ are normalized according to

$$
\begin{aligned}
& \sum_{j_{n} j_{p}}\left(G_{j_{p} j_{n}}^{J i} W_{j_{p} j_{n}}^{J i^{\prime}}\left(1-y_{j_{p}}^{2}-y_{j_{n}}^{2}\right)\right. \\
& \left.\quad-T_{j_{p} j_{n}}^{J i} S_{j_{p} j_{n}}^{J i^{\prime}}\left(y_{j_{p}}^{2}-y_{j_{n}}^{2}\right)\right)=\delta_{i i^{\prime}} /\left(1-\mathrm{e}^{-\omega_{J i} / T}\right) .
\end{aligned}
$$

From the equation of motion (A2) we get the system of TQRPA equations for unknown variables $G, W, T$, and $S$ and phonon energies

$$
\begin{aligned}
& G_{j_{p} j_{n}}^{J i} \pm W_{j_{p} j_{n}}^{J i}=\frac{2 \hat{J}^{-2}}{\varepsilon_{j_{p} j_{n}}^{(+)} \mp \omega_{J i}} \sum_{n=1}^{2 N} d_{j_{p} j_{n}}^{(J n)} \kappa_{1}^{(n)}\left(u_{j_{p} j_{n}}^{(+)} D_{+}^{J i n} \pm u_{j_{p} j_{n}}^{(-)} D_{-}^{J i n}\right) \\
& T_{j_{p} j_{n}}^{J i} \pm S_{j_{p} j_{n}}^{J i}=\frac{2 \hat{J}^{-2}}{\varepsilon_{j_{p} j_{n}}^{(-)} \mp \omega_{J i}} \sum_{n=1}^{2 N} d_{j_{p} j_{n}}^{(J n)} \kappa_{1}^{(n)}\left(v_{j_{p} j_{n}}^{(-)} D_{+}^{J i n} \pm v_{j_{p} j_{n}}^{(+)} D_{-}^{J i n}\right)
\end{aligned}
$$

where

$$
\begin{aligned}
& D_{+}^{J i n}=\sum_{j_{p} j_{n}} d_{j_{p} j_{n}}^{(J n)}\left\{u_{j_{p} j_{n}}^{(+)}\left(1-y_{j_{p}}^{2}-y_{j_{n}}^{2}\right) G_{j_{p} j_{n}}^{J i}-v_{j_{p} j_{n}}^{(-)}\left(y_{j_{p}}^{2}-y_{j_{n}}^{2}\right) T_{j_{p} j_{n}}^{J i}\right\} \\
& D_{-}^{J i n}=\sum_{j_{p} j_{n}} d_{j_{p} j_{n}}^{(J n)}\left\{u_{j_{p} j_{n}}^{(-)}\left(1-y_{j_{p}}^{2}-y_{j_{n}}^{2}\right) W_{j_{p} j_{n}}^{J i}-v_{j_{p} j_{n}}^{(+)}\left(y_{j_{p}}^{2}-y_{j_{n}}^{2}\right) S_{j_{p} j_{n}}^{J i}\right\}
\end{aligned}
$$

In the above equation we have introduced the following linear combination of the Bogoliubov $(u, v)$ coefficients: $u_{j_{p} j_{n}}^{( \pm)}=u_{j_{p}} v_{j_{n}} \pm v_{j_{p}} u_{j_{n}}, v_{j_{p} j_{n}}^{( \pm)}=u_{j_{p}} u_{j_{n}} \pm v_{j_{p}} v_{j_{n}}$. The factors $d_{j_{p} j_{n}}^{(J n)}$ are given by

$$
d_{j_{p} j_{n}}^{(J n)}=\left\{\begin{array}{lc}
f_{j_{p} j_{n}}^{(J k)}, & \text { if } n=k \\
g_{j_{p} j_{n}}^{(J J k)}, & \text { if } n=N+k
\end{array}\right.
$$

for natural parity phonons $\left(\pi=(-1)^{J}\right)$, and

$$
d_{j_{p} j_{n}}^{(J n)}=\left\{\begin{array}{lc}
g_{j_{p} j_{n}}^{(J-1 J k)}, & \text { if } n=k \\
g_{j_{p} j_{n}}^{(J+1 J k)}, & \text { if } n=N+k
\end{array}\right.
$$


for unnatural parity phonons $\left(\pi=(-1)^{J+1}\right)$.

Because of the separable form of the residual interaction the TQRPA equations can be reduced to the set of equations for $D_{\mp}^{J i n}$

$$
\left(\begin{array}{cc}
\mathcal{M}_{1}-\frac{1}{2} I & \mathcal{M}_{2} \\
\mathcal{M}_{2} & \mathcal{M}_{3}-\frac{1}{2} I
\end{array}\right)\left(\begin{array}{c}
D_{+} \\
D_{-}
\end{array}\right)=0 .
$$

The matrix elements of the $2 N \times 2 N$ matrices $\mathcal{M}_{\beta}$ are the following:

$$
\begin{aligned}
& \mathcal{M}_{1,3}^{n n^{\prime}}=\frac{\kappa_{1}^{\left(n^{\prime}\right)}}{\hat{J}^{2}} \sum_{j_{p} j_{n}} d_{j_{p} j_{n}}^{(J n)} d_{j_{p} j_{n}}^{\left(J n^{\prime}\right)}\left\{\frac{\varepsilon_{j_{p} j_{n}}^{(+)}\left(u_{j_{p} j_{n}}^{( \pm)}\right)^{2}}{\left(\varepsilon_{j_{p} j_{n}}^{(+)}\right)^{2}-\omega_{J i}^{2}}\left(1-y_{j_{p}}^{2}-y_{j_{n}}^{2}\right)-\frac{\varepsilon_{j_{p} j_{n}}^{(-)}\left(v_{j_{p} j_{n}}^{(\mp)}\right)^{2}}{\left(\varepsilon_{j_{p} j_{n}}^{(-)}\right)^{2}-\omega_{J i}^{2}}\left(y_{j_{p}}^{2}-y_{j_{n}}^{2}\right)\right\}, \\
& \mathcal{M}_{2}^{n n^{\prime}}=\frac{\kappa_{1}^{\left(n^{\prime}\right)}}{\hat{J}^{2}} \omega_{J i} \sum_{j_{n} j_{p}} d_{j_{p} j_{n}}^{(J n)} d_{j_{p} j_{n}}^{\left(J n^{\prime}\right)}\left\{\frac{u_{j_{p} j_{n}}^{(+)} u_{j_{p} j_{n}}^{(-)}}{\left(\varepsilon_{j_{p} j_{n}}^{(+)}\right)^{2}-\omega_{J i}^{2}}\left(1-y_{j_{p}}^{2}-y_{j_{n}}^{2}\right)-\frac{v_{j_{p} j_{n}}^{(+)} v_{j_{p} j_{n}}^{(-)}}{\left(\varepsilon_{j_{p} j_{n}}^{(-)}\right)^{2}-\omega_{J i}^{2}}\left(y_{j_{p}}^{2}-y_{j_{n}}^{2}\right)\right\},
\end{aligned}
$$

where $1 \leq n, n^{\prime} \leq 2 N$. Thus, the TQRPA eigenvalues $\omega_{J i}$ are the roots of the secular equation

$$
\operatorname{det}\left(\begin{array}{cc}
\mathcal{M}_{1}-\frac{1}{2} I & \mathcal{M}_{2} \\
\mathcal{M}_{2} & \mathcal{M}_{3}-\frac{1}{2} I
\end{array}\right)=0,
$$

while the phonon amplitudes corresponding the TQRPA eigenvalue $\omega_{J i}$ are determined by Eqs. (A5), (A6), and (A8), taking into account the normalization condition (A7).

[1] K. Langanke, and G. Martínez-Pinedo, Rev. Mod. Phys. 75, 819 (2003).

[2] H.-Th. Janka, K. Langanke, A. Marek, G. Martínez-Pinedo, and B. Müller, Phys. Rep. 442, 38 (2007).

[3] E. Caurier, K. Langanke, G. Martínez-Pinedo, and F. Nowacki, Nucl. Phys. A 653, 439 (1999).

[4] K. Langanke and G. Martínez-Pinedo, Nucl. Phys. A 673, 481 (2000).

[5] P. B. Radha, D. J. Dean, S. E. Koonin, K. Langanke, and P. Vogel, Phys. Rev. C 56, 3079 (1997).

[6] G. Wendell Misch, George M. Fuller, and B. Alex Brown, Phys. Rev. C 90, 065808 (2014).

[7] A. A. Dzhioev, A. I. Vdovin, V. Yu. Ponomarev, and J. Wambach, Phys. At. Nucl. 72, 1320 (2009).

[8] Alan A. Dzhioev, A. I. Vdovin, V. Yu. Ponomarev, J. Wambach, K. Langanke, and G. Martínez-Pinedo, Phys. Rev. C 81, 015804 (2010). 
[9] J. Cooperstein, and J. Wambach, Nucl. Phys. A 420, 591 (1984).

[10] Alan A. Dzhioev, A. I. Vdovin, J. Wambach, and V. Yu. Ponomarev, Phys. Rev. C 89, 035805 (2014).

[11] Alan A. Dzhioev, A. I. Vdovin, and J. Wambach, Phys. Rev. C 92, 045804 (2015).

[12] V. G. Soloviev, Theory of atomic nuclei: Quasiparticles and phonons (Taylor \& Francis, 1992).

[13] Nguyen Van Giai, Ch. Stoyanov, and V. V. Voronov, Phys. Rev. C 57, 1204 (1998).

[14] A. P. Severyukhin, Ch. Stoyanov, V. V. Voronov, Nguyen Van Giai, Phys. Rev. C 66, 034304 (2002).

[15] A. P. Severyukhin, V. V. Voronov, and Nguyen Van Giai, Prog. Theor. Phys. 128, 489 (2012).

[16] N. Paar, G. Colò, E. Khan, and D. Vretenar, Phys. Rev. C 80, 055801 (2009).

[17] A. F. Fantina, E. Khan, G. Colò, N. Paar, and D. Vretenar, Phys. Rev. C 86, 035805 (2012).

[18] Y. F.Niu, N. Paar, D. Vretenar, and J. Meng, Phys. Rev. C 83, 045807 (2011).

[19] J. Wambach, in Weak and Electromagnetic Interactions in Nuclei, ed. H. V. Klapdor (Springer, Heidelberg, 1986), p. 950.

[20] H. Umezawa, H. Matsumoto, and M. Tachiki, Thermo field dynamics and condensed states, (North-Holland Pub. Co., 1982).

[21] Y. Takahashi and H. Umezawa, Int. J. Mod. Phys. B 10, 1755 (1996).

[22] I. Ojima, Annals Phys. 137, 1 (1981).

[23] Alan A. Dzhioev and A. I. Vdovin, Int. J. Mod. Phys. E 18, 1535 (2009).

[24] P.F. Bortignon, A. Bracco, and R. A. Broglia, Giant Resonances: Nuclear Structure at Finite Temperature, Contemporary Concepts in Physics (Harwood, 1998).

[25] G.F. Bertsch and S.F. Tsai, Phys. Rep. 18, 125 (1975).

[26] Nguyen Van Giai and H. Sagawa, Phys. Lett. B 106, 379 (1981).

[27] A. L. Goodman, Nucl. Phys. A 352, 30 (1981).

[28] O. Civitarese, G. Dussel, and R. P. J. Perazzo, Nucl. Phys. A bf 404, 15 (1983).

[29] E. Chabanat, P. Bonche, P. Haensel, J. Meyer, and R. Schaeffer, Nucl. Phys. A 635, 231 (1998).

[30] J. Bartel, P. Quentin, M. Brack, C. Guet and H.-B. Håkansson, Nucl. Phys. A 386, 79 (1982).

[31] S. El-Kateb, K. P. Jackson, W. P. Alford, R. Abegg, R. E. Azuma, B. A. Brown, et al., Phys. Rev. C 49, 3128 (1994).

[32] V. A. Kuzmin and V. G. Soloviev, J. Phys. G: Nucl. Phys. 10, 1507 (1984). 
[33] A. P. Severyukhin, V. V. Voronov, I. N. Borzov, and N. V. Giai, Romanian J. Phys. 581048 (2013).

[34] P. Sarriguren, Phys. Rev. C 87, 045801 (2013). 


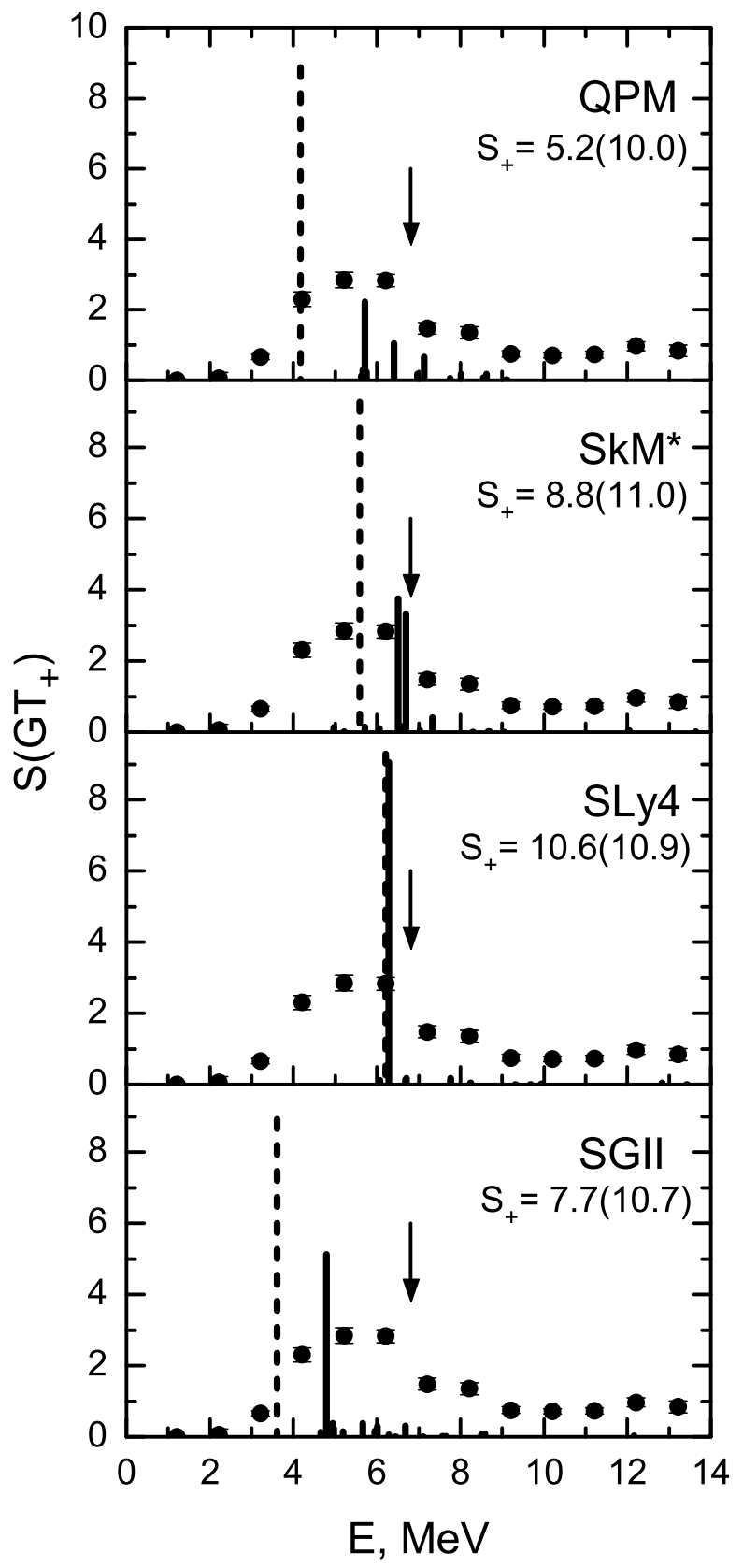

FIG. 1. Ground state GT + strength distributions in ${ }^{56} \mathrm{Fe}$ calculated with the SGII, SLy4 and SkM* forces. The excitation energies are related to the parent ground state. For comparison, the $\mathrm{GT}_{+}$ strength calculated with the QPM Hamiltonian is also shown [8]. The solid peaks denote the QRPA results, and the dashed peaks represent the unperturbed BCS distributions calculated neglecting the residual p-h interaction. The total $\mathrm{GT}_{+}$strength is denoted by $S_{+}$and the unperturbed values of $S_{+}$are given in parentheses. Experimental data [31] are displayed by points and for clearer presentation they are multiplied by a factor of 5 . The $\mathrm{GT}_{+}$centroid energy from the LSSM calculation [3] is indicated by an arrow. 


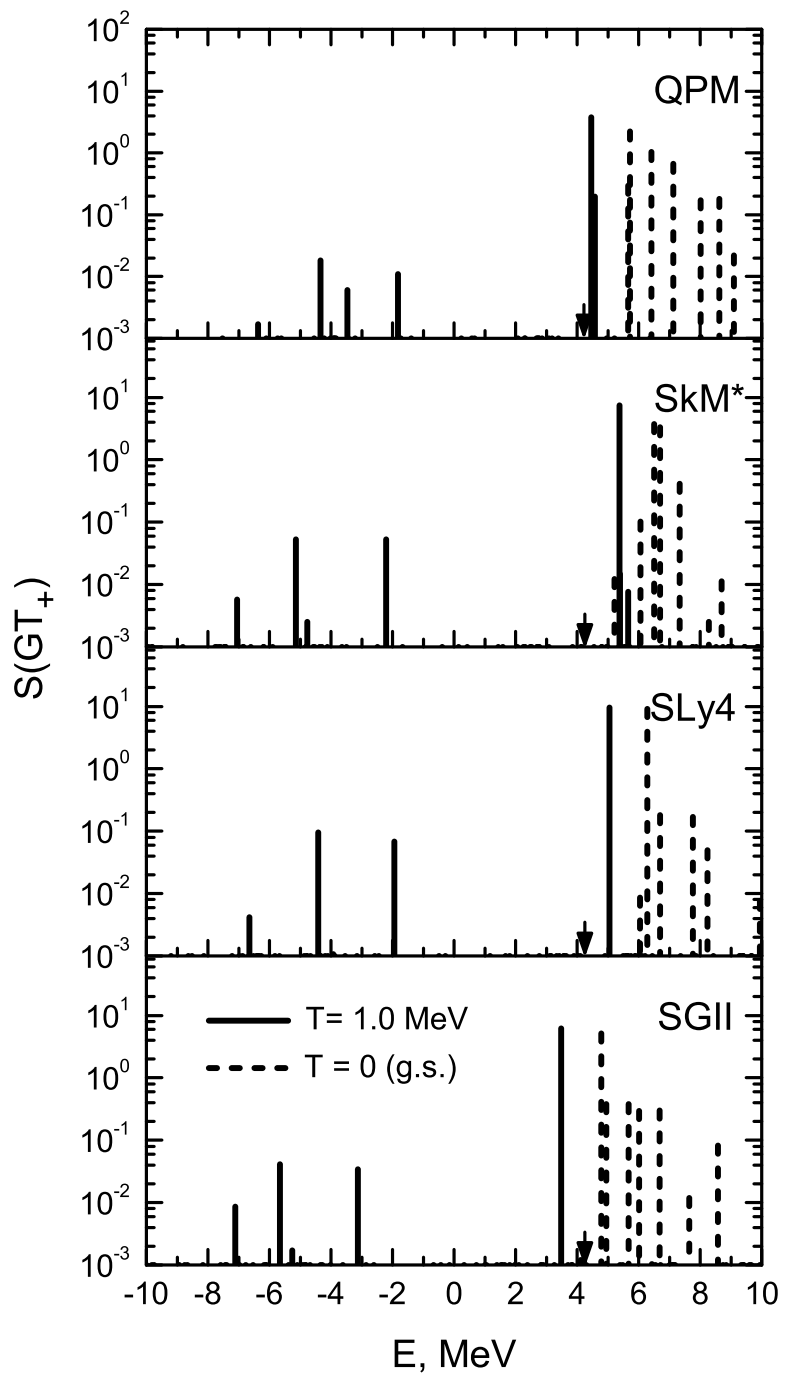

FIG. 2. GT + strength functions for ${ }^{56} \mathrm{Fe}$ calculated at $T=0$ (dashed peaks) and $T=1.0 \mathrm{MeV}$ (solid peaks). The arrows indicate the ground-state reaction threshold for the electron capture $(Q=4.21 \mathrm{MeV})$ 

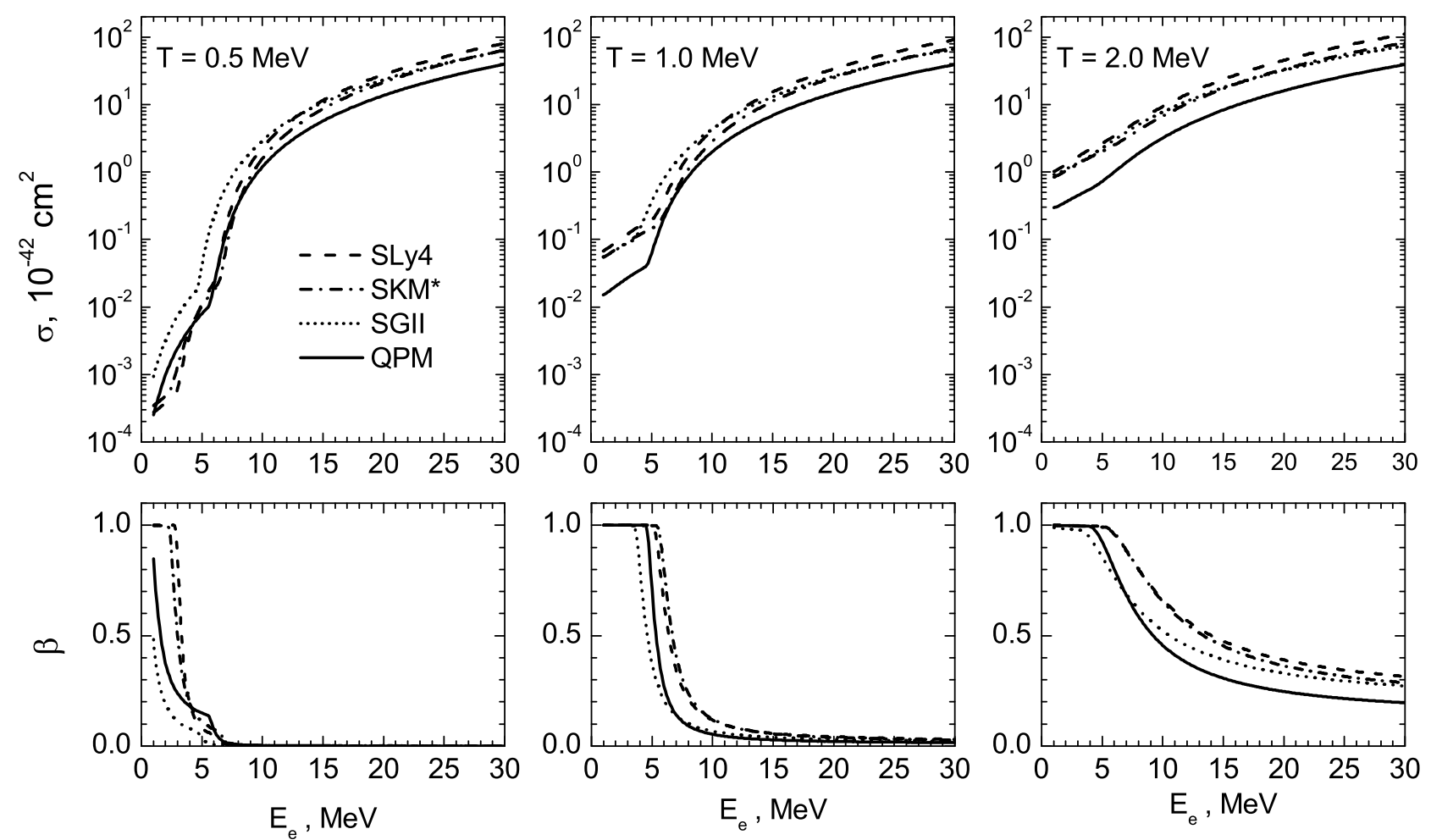

FIG. 3. Top panels: Electron capture cross sections for ${ }^{56} \mathrm{Fe}$ for three different temperatures. The Skyrme-QRPA results are compared with those obtained by the QPM-TQRPA calculations. Bottom panels: Temperature dependence of the ratio $\beta\left(E_{e}, T\right)$ of the exoergic electron absorption to the reaction cross section. 

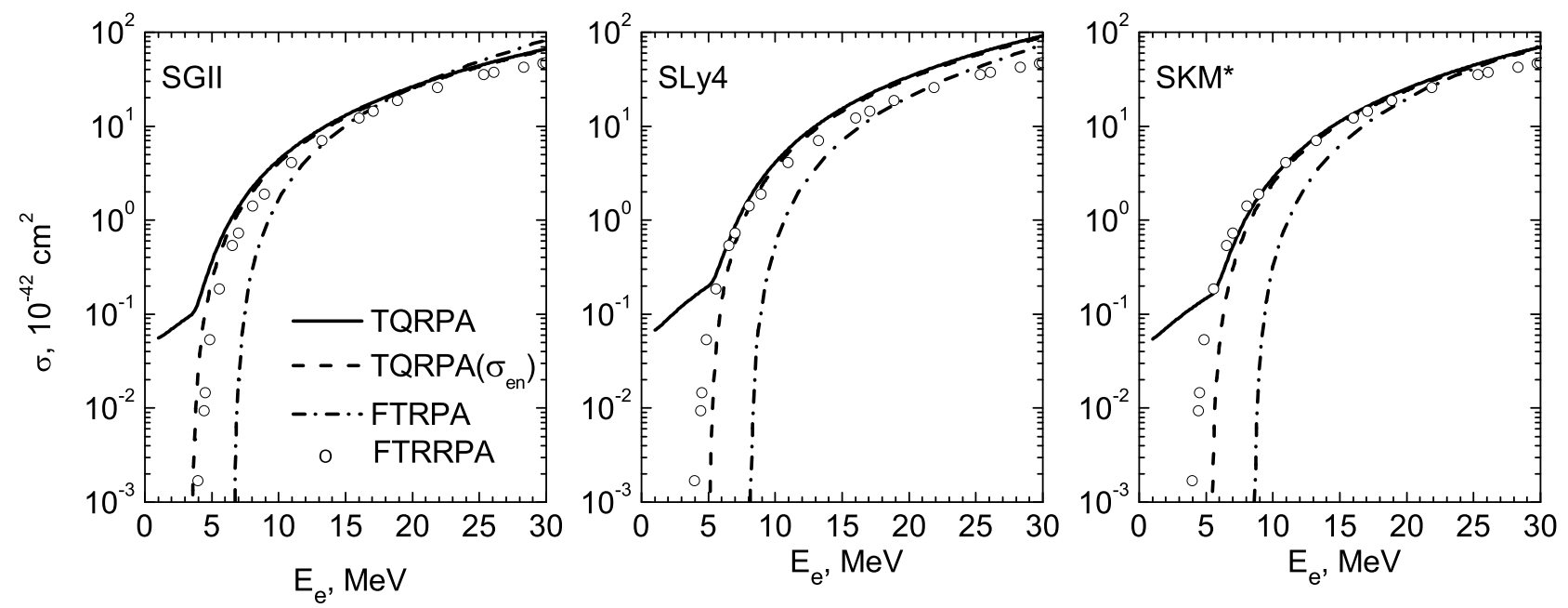

FIG. 4. Electron capture cross sections for ${ }^{56} \mathrm{Fe}$ at $T=1.0 \mathrm{MeV}$. The Skyrme-TQRPA results are compared with the cross sections calculated within the FTRPA [17] and the FTRRPA [18] framework 